\title{
Numerical Experiments of Pore Scale for Electrical Properties of Saturated Digital Rock
}

\author{
Wenzheng Yue ${ }^{1}$, Guo Tao' ${ }^{1}$ Xiaochuan Zheng ${ }^{2}$, Ning Luo ${ }^{2}$ \\ ${ }^{1}$ State Key Laboratory of Petroleum Resource and Prospecting, Key Laboratory of Earth Prospecting and Information \\ Technology, CNPC Key Lab of Well Logging, China University of Petroleum, Beijing, China \\ ${ }^{2}$ Sichuan Petroleum Administration Logging Company Ltd., CNPC, Chongqing, China \\ E-mail: yuejack1@sina.com \\ Received February 17, 2011; revised April 1, 2011; accepted May 2, 2011
}

\begin{abstract}
The two dimensional Lattice Gas Automation (LGA) was applied to simulate the current flow in saturated digital rock for revealing the effects of micro structure and saturation on the electrical transport properties. The digital rock involved in this research can be constructed by the pile of matrix grain with radius obtained from the SEM images of rock sections. We further investigate the non-Archie phenomenon with the LGA and compare micro-scale numerical modeling with laboratory measurements. Based on results, a more general model has been developed for reservoir evaluation of saturation with higher accuracy in oilfield application. The calculations from the new equation show very good agreement with laboratory measurements and published data on sandstone samples.
\end{abstract}

Keywords: Lattice Gas Automation, Digital Rock, Non-Archie Phenomenon, Water Saturation

\section{Introduction}

The electrical transport properties of saturated rock are fundament of constructing a relation between resistivity and water saturation in petrophysics [1-3]. The physical experiment of rock is a main approach to research the electrical transport properties for revealing the effects of pore structure and fluids distribution on the whole resistivity. Based on the results of experiments, many models of reservoir evaluation have been developed in the past decades for exploration of oil and gas in petroleum industry [1-4]. As pointed by Batchelor [5], the resistivity of rock is affected by not only the electrical transport properties of each component but also the structure of distribution.

However, due to the limitations of laboratory experiments as the approach of macro scale, the micro pore structure, the flow of fluid and electrical current in a rock cannot be directly observed and controlled. Thus, it is not possible to quantify the factors that influence the relation between resistivity and physical parameters of rock such as porosity and water saturation. Therefore, many researchers have tried to simulate the behavior numerically at the micro scale [6-9].

LGA method, being developed originally for fluid dy- namics, was spread rapidly into numerical simulation of many fields in the last decades and believed to permit accurate simulation of fluids flow at the microscopic scale for grossly irregular geometries [10-13]. LGA has been successfully applied to model fluid and current flow in saturated porous media to calculate the effective electrical conductivity of single-phase fluid-saturated porous media as a function of porosity and conductivity ratio between the pore-filling fluid and the solid matrix for various microscopic structures of the pore space.

In this paper, the LGA method was applied to simulate the current flow in digital rock saturated with fluids for understanding the mechanism of how the micro factors affecting the whole resistivity of rock at pore scale.

\section{Lattice Gas Automation for Simulation of Current Flow}

\subsection{Lattice Gas Automation}

The LGA method was developed in 1976 named HPP [14] by improving the conventional cellular automation method, which is absence of rotational symmetry for its rectangular lattice space. In 1986, the FHP model [10] with the hexagon lattice has overcome the drawbacks of 
HPP and retrieved the Navier-Stokes (NS) equation at macro scale. Latter, the improvement of FHP by introducing the rest particles makes the LGA method more reasonable for simulation of fluid flow.

In the LGA, the fluid, space and time are all discrete. The fluid is taken as a collection of small particles with only unit mass and without volume. The particles are distributed in the node of discrete space, moving forward along the lattice and following local interactions rules. The Pauli principle is applied to control the distribution and evolution of the particles among the nodes. At each time step, particles first advance one lattice unit to a neighbouring site in the direction they were headed. Then they may undergo collisions that must conserve mass and momentum, so that the continuity and momentum conservation conditions for incompressible fluids are locally satisfied. For the LGA, the collision and streaming of particles can be described by the evolution of the distribution function of particle density as following

$$
f_{i}(x+e, t+1)=f_{i}(x, t)+\Omega(f(x, t)) .
$$

where, the $f_{i}(x, t)$ is the distribution function of particle density at lattice node $x$, time $t$, moving along the direction $i$ with the velocity of $e_{i}$. The $\Omega$ is the collision rules such as shown in Figure 1 to control the redistribution of particles.

Based on the collision rules in the LGA, the NS equation can be retrieved with the definition of density and momentum to be

$$
\begin{aligned}
& \text { Density: } \rho=\sum_{i} f_{i}(x, t), \\
& \text { Momentum: } \rho u=\sum_{i} e_{i} \cdot f_{i} .
\end{aligned}
$$

For an uncompressible flow, the NS equation can reduce to Darcy's law. By comparing Darcy's law with Ohm's law, we can find both the two formulas being of the same mathematical expression if the electric charges replace the fluid particles and the electric potential replace the pressure. The analog between the Darcy's and Ohm's law demonstrates that it is reasonable to simulate the electric current flow in porous media with the LGA method.

\subsection{Simulation of Current with the LGA}

To verify the reliable of LGA in simulation of current flow, the LGA method was used to obtain the conductivity of binary mixtures in the parallel and serial mode [15]. The parallel or serial modes of mixtures mean the alternating layers of conductors are parallel or perpendicular to the direction of flow respectively as that shown in Figure 2.

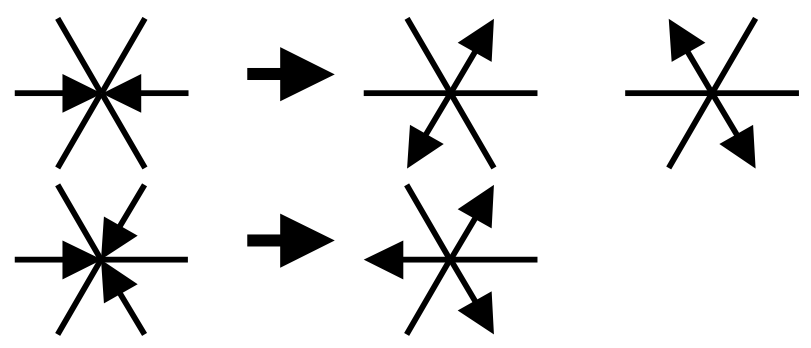

Figure 1. Collision rules of FHP model.
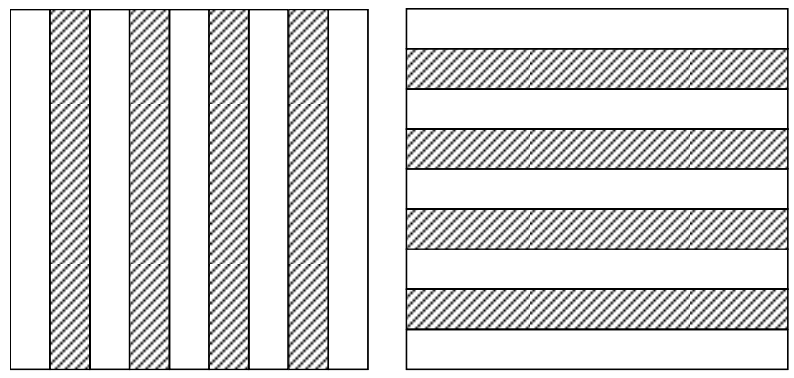

Figure 2. Binary mixtures in the parallel and serial mode respectively.

The theoretical value of the conductivity of binary mixtures in the parallel and serial modes can be calculated by the formulas as fellow:

$$
\begin{gathered}
\sigma_{p}=\sigma_{1} \phi_{1}+\sigma_{1} \phi_{2}, \\
\frac{1}{\sigma_{s}}=\frac{\phi_{1}}{\sigma_{1}}+\frac{\phi_{2}}{\sigma_{2}} .
\end{gathered}
$$

where, $\phi_{1}$ and $\phi_{2}$ are defined as the volume fractions of two components, $\sigma_{1}$ and $\sigma_{2}$ are defined as the conductivity of phase 1 and 2 in mixtures, $\sigma_{p}$ and $\sigma_{s}$ are the whole effective conductivity of binary mixtures in the parallel and serial mode respectively. It is believed that Equations (4) and (5) give the lower and upper limits for the effective conductivity of binary mixtures.

The simulated results of the LGA with models of binary mixtures in the parallel and serial mode have been plotted in Figure 3. The upper line is the theoretical value of parallel models, and the lower is the theoretical value of serial models respectively. As a comparison, the corresponding theoretical value calculated with a ratio of two conductive phases $\sigma_{2} / \sigma_{1}$ being 0.04 has been shown in Figure 3 together. It is clear that the conductivity calculated with the LGA is in good agreement with the theoretical value for binary mixtures in the parallel mode. However, the simulated results obtained by the LGA are little higher than theoretical values for models in the serial mode, especially when the volume fraction of high conductive phase being obviously larger than that of low conductive phase, which is caused by contribution of low conductive phase to the whole conductivity in the LGA 


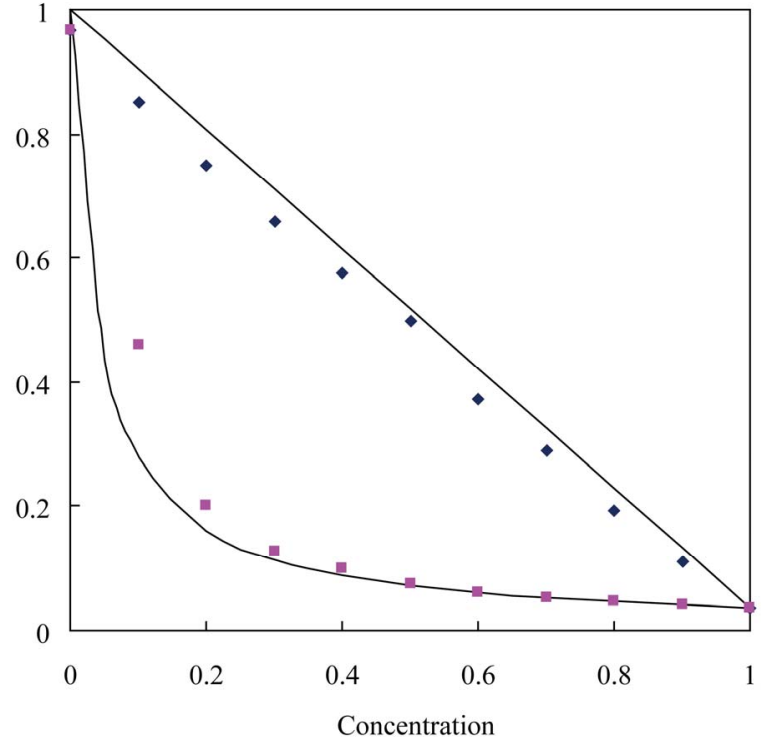

Figure 3. Comparison of results by the LGA with that by theoretical model [15].

being less than theoretical value of the serial mode. From Figure 3, the validity of the LGA in simulation of electrical transport has been demonstrated by the good agreement of comparison between simulated results and theoretical value.

\section{Digital Rock Model}

In petrophysics, the research has been increasingly focused on how the factors of pore scale influencing the electrical transport properties of saturated rock. As mentioned above, due to the limitations of laboratory experiments as the approach of macro scale, it is not possible to quantify the factors of pore scale that influence the relation between resistivity and physical parameters of rock and filling fluids. Therefore, as a complement to physical experiments, the digital rock is proved to be an effective numerical method to investigate the physical transport properties at the micro scale $[9,16,17]$.

The digital rock involved in this research is constructed by the pile of matrix grain. The distribution of radius of matrix grain can be obtained from the 2D SEM images of rock sections, by the technique of digital image processing. Figure 4 shows the distribution of matrix grain obtained from a SEM image of rock. The horizontal axis is the value of radius, and the vertical axis is the percentage of matrix grain. In Figure 4, it is clear that the most of radius of matrix grain is in a range from $7.35 \mu \mathrm{m}$ to $735 \mu \mathrm{m}$, and only a very few of radius of matrix grain is less than $0.735 \mu \mathrm{m}$.

According the obtained information, the matrix grains will be deposit originally into a regular space through pilling up of particles to construct an unconsolidated digital rock. Consequently, the deposited matrix grain is consolidated by compacting under pressure. During the compaction, the grains may be moved from the original positions to the new sites for reaching the balance of contacted particles as shown in Figure 5. Figure 5 shows an enlarged picture of a small portion of thus constructed digital rock. In Figure 5, the radius of matrix grain used to construct digital rock is different with each other, and the distribution of radius is identical with that shown in Figure 4.

Generally, the micro structure of pore in rock is dependent on the form of pilling up and shapes of matrix

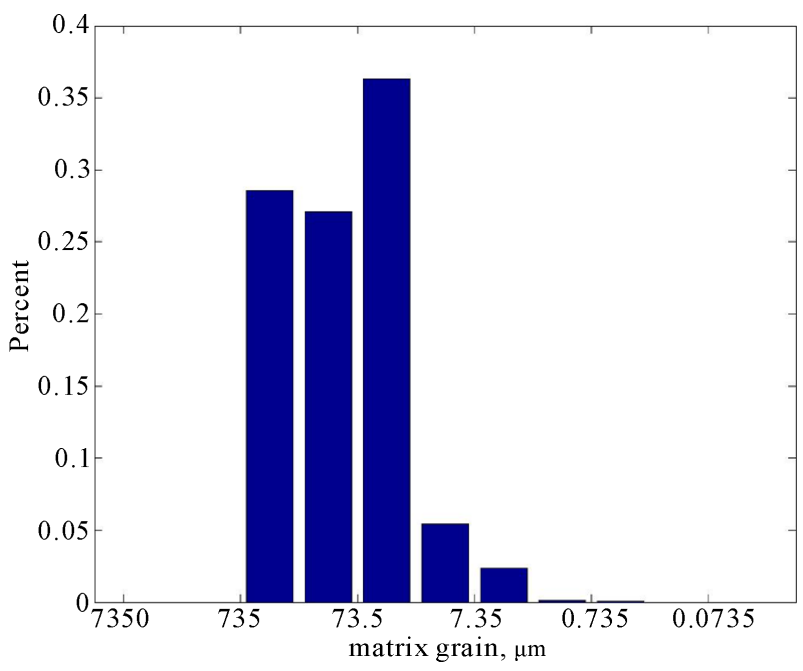

Figure 4. The distribution of matrix grain obtained from a SEM image of rock.

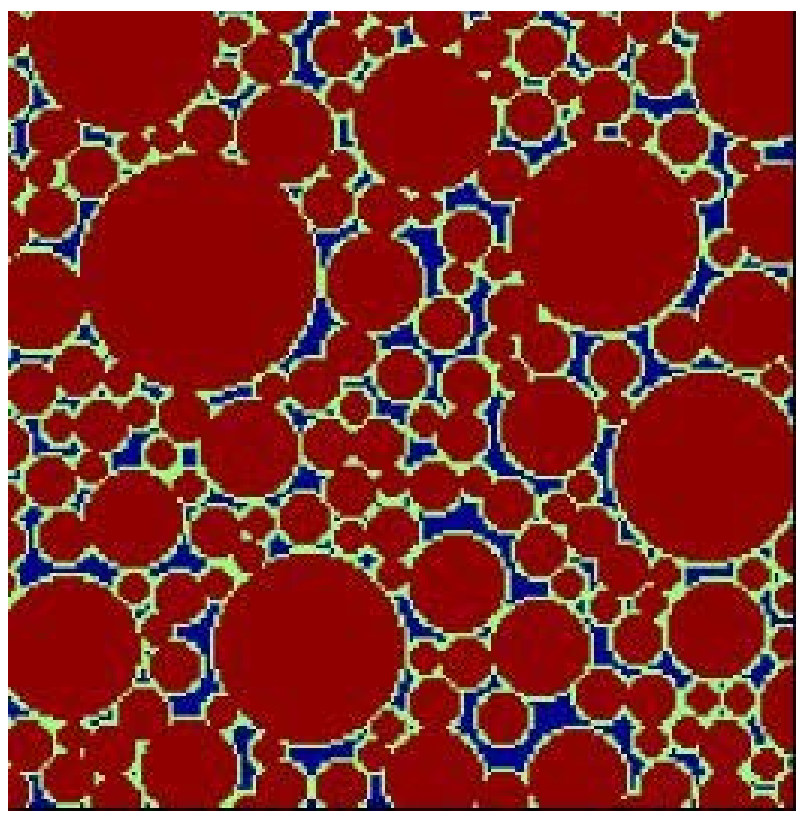

Figure 5. The digital rock of sphere grain. 
grain. More complex of the matrix grain used to construct digital rock will result in more complex of the micro structure in pore. Therefore, it is essential to use various grain geometries to construct the digital core sample for revealing the effects of the micro pore structure on the electrical transport properties of porous rock. In this research, the main shapes of matrix grain, employed to construct digital rock for investigating the effects of the micro structure, are randomly distributed point, triangle, diamond-shaped, apparent rectangular and grain cluster. The digital rock constructed in this way can permits the modeling of current flow in grossly irregular pore space geometries, with variation of the porosity as large as desired. Finally, the constructed digital rock should be saturated with water and oil in pore space to research the effects of saturation and distribution of fluids on the whole electrical properties of rock.

\section{Simulated Results and Discusses}

During the simulation of LGA for electrical transport properties, the method proposed by Rothman was used to apply the electrical voltages on the digital rock sample. The upper and lower boundaries of the sample are non-slip solid boundary in the LGA for implementing the insulation boundaries. The periodic boundary on the left and right boundaries of the sample is used to simulate the infinite boundaries in the horizontal direction.

In 1942, Archie [1] proposed a quantitative formula to relate the resistivity index $(I)$, defined as ratio of resistivity of rock saturated with oil and water $\left(R_{t}\right)$ to that of rock saturated with saline water $\left(R_{0}\right)$, to the water saturation $\left(S_{w}\right)$ as

$$
I=\frac{R_{t}}{R_{o}}=\frac{b}{S_{w}^{n}},
$$

where $b$ is a real parameter, $n$ is usually called the saturation exponent. Equation (6) indicates the relation between resistivity index and water saturation $\left(I-S_{w}\right)$ will be linear in log-log scale as shown in Figure 6 with $b \approx 1$ and $n \approx 2$ in the most cases of pure sand stones.

The transport of current in saturated digital rock has been simulated by the LGA to obtain the resistivity of sample with different saturation in pore space. The resistivity index can be calculated with the definition in Equation (6). The calculated parameters of $I-S_{w}$ relation for the digital rocks with the same porosity are given in Table 1. It can be seen from Table 1 that the micro structures of pore will affect the $I-S w$ relation. The parameter $n$ of diamond-shaped grain of matrix is large than that of triangle grain, and the smallest $n$ is obtained from the digital rock with the rectangle grain of matrix.

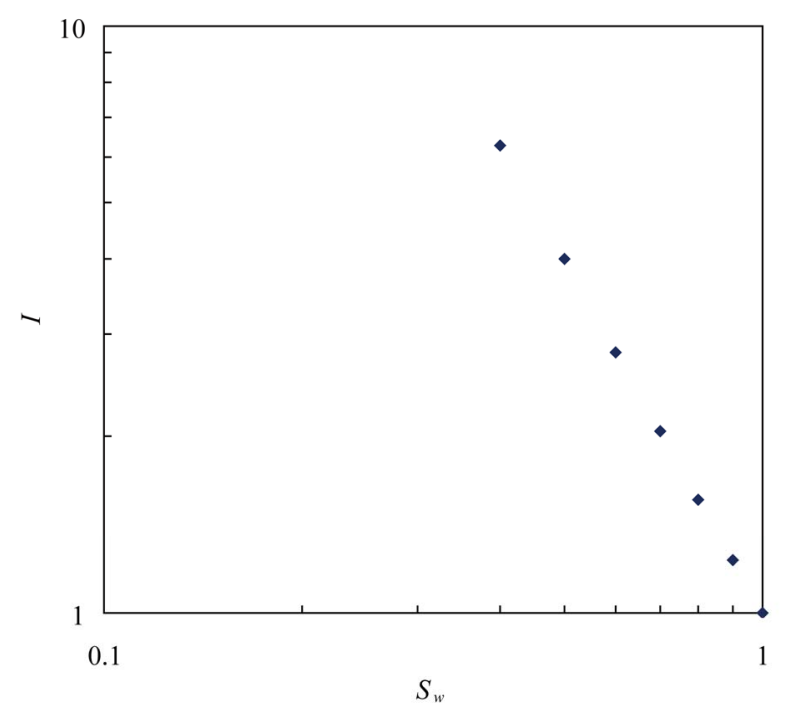

Figure 6. $I-S_{w}$ relation of archie.

Table 1. Parameters $\boldsymbol{b}$ and $\boldsymbol{n}$ for different matrix grain.

\begin{tabular}{ccc}
\hline Shapes of matrix grain & $b$ & $n$ \\
\hline Diamod & 0.9649 & 1.4115 \\
Triangle & 0.9868 & 1.3543 \\
Randomly point & 0.9854 & 1.2413 \\
Rectangle & 1.0632 & 1.1717 \\
\hline
\end{tabular}

In the past decades, the nonlinear relation of $I-S w$ in log-log coordinates, called non-Archie phenomenon, has been increasingly observed from the experiments $[3,8$, 18-20]. Many researches have been done to explain the mechanism of non-Archie phenomena through laboratory experiments of rock. However, due to the limitations of laboratory experiments as the approach of macro scale, the micro pore structure, the flow of fluid and electrical current in a rock cannot be directly observed and controlled $[21,22]$. Thus, it is not possible to quantify the factors that influence the relation between resistivity index and physical parameters of rock such as water saturation.

During the simulation of LGA, it can be seen that the non-Archie relation of $I-S_{w}$ will become a generally phenomenon when water saturation being lower than a critical value. Without losing the generality, one group data has been plotted in Figure 7 to show clearly the nonArchie relation of $I-S w$.

In Figure 7, the horizontal axis is the value of water saturation, and the vertical axis is the value resistivity index. It can be seen that the critical value of water saturation is 0.55 . The Archie relation of $I-S_{w}$ is broken obviously when water saturation being lower than the criti- 


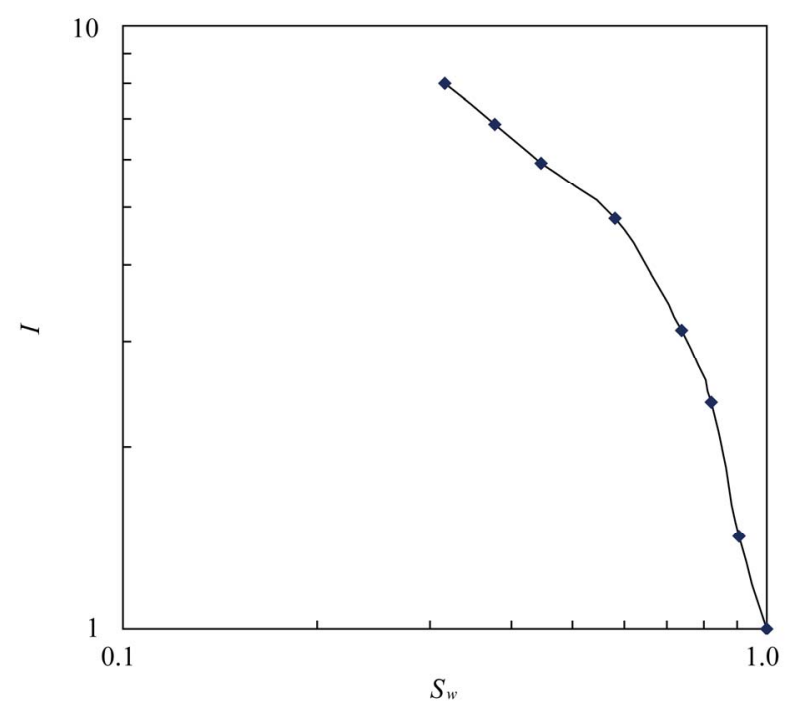

Figure 7. The simulated relation of $I-S_{w}$.

cal value. The non-Archie behavior of $I-S_{w}$ relation will become prominent when $S_{w}$ being lower than 0.55. Clearly, the $I-S_{w}$ relationship in a log-log scale drops off towards the saturation axis as $S_{w}$ decreases. This means that the parameter $n$ would decrease with the increasing of $S_{w}$. This non-Archie behavior can be explained as due to the effects of matrix- pore fluids network conducting.

\section{A New Formula for the Non-Archie $I-S_{w}$}

Both the simulated results of LGA and the measurements of laboratory experiments by many others, indicate that the relation of $I I-S_{w}$ is not a linear in log-log scale as described by Archie's equation. Generally, the $I-S_{w}$ relationships in a log-log scale drops off towards the saturation axis as $S_{w}$ decreases, which means that the Archie exponent $n$ is not a constant but a function of $S_{w}$ itself. A reasonable interpretation for this phenomenon is that the saturation exponent $n$ is the measure of the changing rate of the current path complexity with respect to saturation and porosity. Hence, a new non-Archie relation of $I-S_{w}$ based on our simulations and observations has been developed to relate $I$ to $S_{w}$ as

$$
I=S_{w}^{-Y S_{w}^{U}}
$$

where $Y$ and $U$ are the parameters related to the pore structure and porosity.

To verify the validity of the Equation (7) in practical application, the formula is applied to process the data published by Diederix which were obtained by wellcontrolled experiments on the artificial rock consisting of smoothed glass beads. The data of Diederix have been plotted in Figure 8. Apparently, the non-Archie $I-S w$ relation can be observed obviously in Figure 8 from these data. The critical value of $S w$ for the non-Archie phenomenon of $I-S w$ relation is 0.45 . The results calculated with Equation (7) have been shown in Figure 8 for comparison. Clearly, it can be seen that the calculated results of Equation (7) fits the laboratory measurements very well. The good agreement between the calculated results and the published data further confirm the validity of Equation (7) in application of processing measured data.

In Figure 8, the diamond-shaped data points are from Diederix on the artificial samples consisting of smoothed glass beads.

The data obtained from the experiments on real rock have been used to further verify the applicability of Equation (7). The measured results and the calculated results with Equation (7) and Archie's Equation are shown in Figure 9 for comparison. In Figure 9, the solid circles

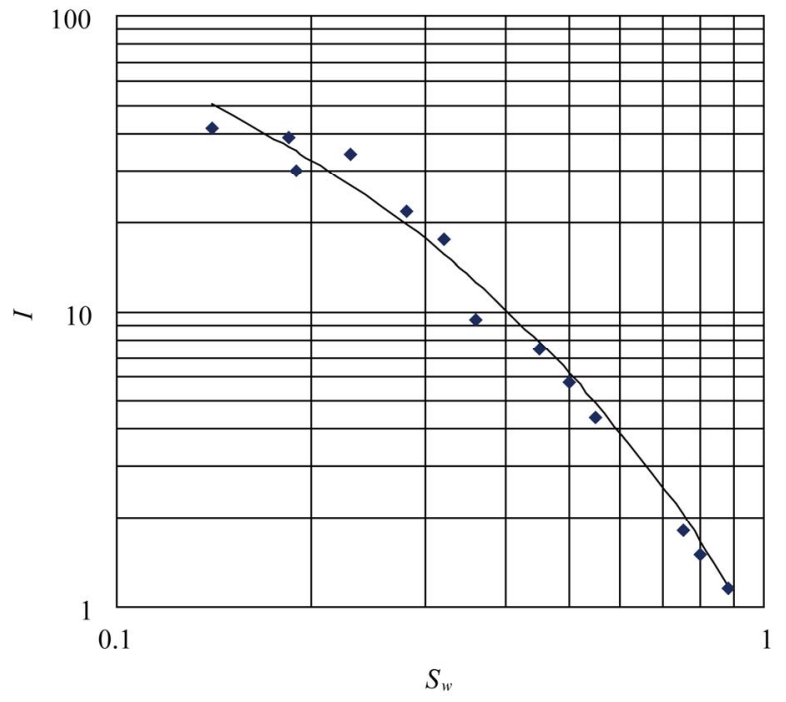

Figure 8. The $I-S_{w}$ relation of artificial rock.

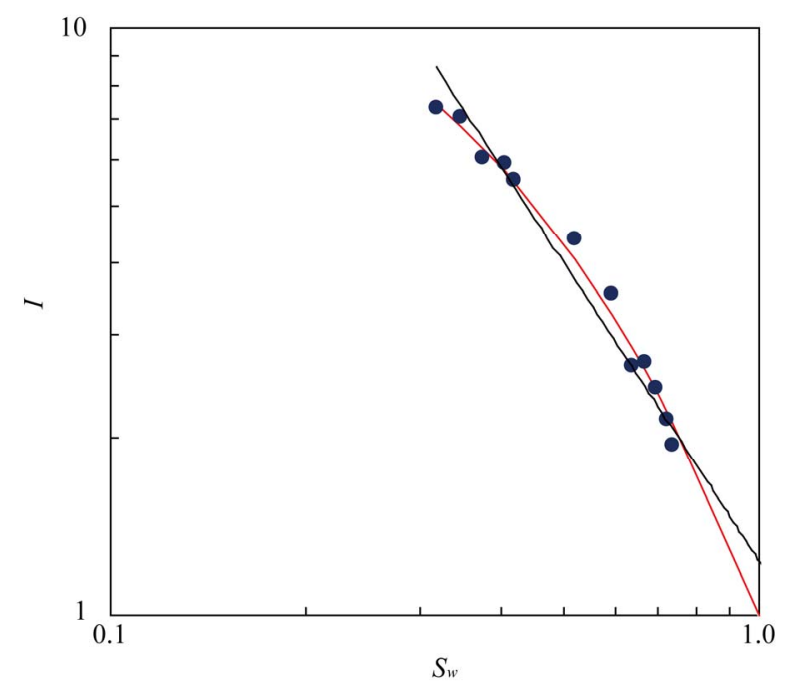

Figure 9. The $I-S_{w}$ relation of rock sample. 
are the data obtained from experiments. And the straight line is the result by fitting the data with the Archie's equation. The smooth curve in red is the results by fitting the data with the new formula.

Clearly, the non-Archie phenomenon of the I-Sw relation can be observed obviously in Figure 9 from these data. The critical value of Sw for the non-Archie phenomenon of the $I-S w$ relation is 0.5 . By comparison, the calculated result using Equation (7) is better agreement with the laboratory measurement than that using Archie's equation. It can be understood that considerable errors could be introduced by processing such data of nonArchie behavior with Archie's equation in reservoir evaluation. The new formula may result in a more accurate evaluation of fluid saturation, especially in the cases of obvious non-Archie phenomenon observed.

\section{Conclusions}

The digital rock involved in this research can be constructed by the pile of matrix grain with radius obtained from the 2D SEM images of rock sections. The LGA method was used to simulate the flow of current in saturated digital rock for revealing the effects of micro structure and water saturation on the electrical transport properties. Based on the simulated results, it is clearly that the saturation exponent $n$ is not constant as described by Archie but is a function of pore structure and fluid saturation. The reasonable interpretation for this phenomenon is that the saturation exponent $n$ is the measure of the changing rate of the current path complexity with respect to saturation and porosity. Moreover, it is understood that the mechanism of non-Archie phenomenon of $I-S w$ relation is due to the inherent nature of the complex current paths of the mixture of matrix and filling fluids in pore space. Therefore, a new equation for reservoir evaluation of saturation has been developed based on the results. The new equation can be applied to calculate the water saturation in reservoir with higher accuracy, especially in the cases of obvious non-Archie phenomenon observed. The calculated results using Equation (7) have been compared with laboratory measurements on real rock core from an oilfield and with data from published papers and have shown very good agreement.

\section{Acknowledgements}

The authors wish to thank the National Natural Science Foundation of China for sponsoring this work under Project No.41074103, and the National Key Fundamental R\&D Plan under Project No. 2007CB209601.

\section{References}

[1] G. E. Archie, "The Electrical Resistivity Log as an Aid in Determining some Reservoir Characteristics," Transaction of American Institute of Mining, Metallurgical, and Petroleum Engineers, Vol. 146, 1942, pp. 54-62.

[2] X. D. Jing, A. Gillesple and B. M. Trewin, "Resistivity Index from Non-Equilibrium Measurements Using Detailed In-situ Saturation Monitoring," SPE Offshore European Conference, Aberdeen, 7-10 September 1993, pp. 456-464.

[3] P. F. Worthington, "Quality Assurance of the Evaluation of Hydrocarbon Saturation from Resistivity Data," SPE Annual Technical Conference and Exhibition, San Antonio, 24-27 September 2006. pp. 1-16. doi:10.2118/103075-MS

[4] N. Li, "Generalised Resistivity-Porosity and Resistivity-Oil Saturation Relationships," Chinese Journal of Geophysics, in Chinese, Vol. 32, No. 5, 1989, pp. 580-591.

[5] G. K. Batchelor, "Transport Properties of Two-Phase Materials with Random Structure," Annual Review of Fluid Mechanics, Vol. 6, 1974, pp. 227-255. doi:10.1146/annurev.f1.06.010174.001303

[6] D. P. Yale, "Network Modeling of Flow Storage and Deformation in Porous Rocks," Ph.D. Thesis, Stanford University, Palo Alto, 1984.

[7] G. Tao, "Elastic and Transport Properties of some Sandstones," Ph.D. Thesis, University of London, London, 1992.

[8] H. N. Man and X. D. Jing, "Network Modeling of Strong and Intermediate Wettability on Electrical Resistivity and Capillary Pressure," Advances in Water Resource, Vol. 24, No. 3-4, 2001, pp. 345-363. doi:10.1016/S0309-1708(00)00061-0

[9] P. E. Oren and S. Bakke, "Process Based Reconstruction of Sandstones and Prediction of Transport Properties," Transport in Porous Media, Vol. 46, No. 2-3, 2002 pp. 311-343. doi:10.1023/A:1015031122338

[10] U. Frisch, B. Hasslacher and Y. Pomeau, "Lattice Gas Automation for the Navier-Stokes Equation," Physical Review Letters, Vol. 56, No. 14, 1986, pp. 1505-1507. doi:10.1103/PhysRevLett.56.1505

[11] D. Rothman, "Cellular Automation Fluids: A Model for Flow in Porous Media," Geophysics, Vol. 53, No. 4, 1988, pp. 509-518. doi:10.1190/1.1442482

[12] J. X. Hu and Y. M. Li, "Modeling Research for Simulating Seismic Wave Propagation in Solid by Cellular Automata," Chinese Journal of Geophysics, in Chinese, Vol. 40, No. 1, 1997, pp. 120-125.

[13] Z. L. Wang and Y. M. Li, "Parallel Algorithm for Simulating Seismic Wave Propagation by Cellular Automata," Chinese Journal of Geophysics, in Chinese, Vol. 42, No. 3, 1999, pp. 410-415.

[14] J. Hardy, "Time Evolution of a Two-Dimentional Model System," Journal of Mathematical Physics, Vol. 14, No. 12, 1973, pp. 1746-1759. doi:10.1063/1.1666248 
[15] M. Küntz, J. C. Mareschal, et al., "Numerical Estimation of Effective Conductivity of Heterogeneous Media with a 2D Cellular Automaton Fluid," Geophysical Research Letters, Vol. 24, No. 22, 1997, pp. 2865-2868. doi:10.1029/97GL52856

[16] O. Hiroshi and M. J. Blunt, "Pore Space Reconstruction Using Multiple-Point Statistics," Journal of Petroleum Science and Engineering, Vol. 46, No. 1-2, 2005, pp. 121-137. doi:10.1016/j.petrol.2004.08.002

[17] M. Pilotti, "Reconstruction of Clastic Porous Media," Transport in Porous Media, Vol. 41, No. 3, 2000, pp. 359-364. doi:10.1023/A:1006696301805

[18] K. M. Diederix, et al., "Anomalous Relationships between Resistivity Index and Water Saturations in the Rotliegend Sandstone," SPWLA 23rd Annual Logging Symposium, Corpus Christi, 6-9 July 1982, pp. 1-16.

[19] X. D. Li, J. Yu and M. Li, "Theoretic Research of Reservoir Rock with Non-Archie Characteristics," Oil-Gas Field
Surface Engineering, in Chinese, Vol. 27, No. 1, 2008, pp. 32-33.

[20] A. U. Al-Kaabi, K. Mimoune and H. Y. Al-Yousef, "Effect of Hysteresis on the Archie Saturation Exponent," SPE Middle East Oil Conference and Exhibition, Manama, 15-18 March 1997, pp. 497-503

[21] D. C. Herrick and W. D. Kennedy, "Electrical Efficiency-A Pore Geometric Theory for Interpreting the Electrical Properties of Reservoir Rocks," Geophysics, Vol. 59, No. 6, 1994, pp. 918-927. doi:10.1190/1.1443651

[22] M. E. Kutay, et al., "Computational and Experimental Evaluation of Hydraulic Conductivity Anisotropy in Hot-Mix Asphalt," International Journal of Pavement Engineering, Vol. 8, No. 1, 2007, pp. 29-43. doi:10.1080/10298430600819147 